\title{
High resolution micro-endoscope: new technology for the early detection and control of bladder
} cancer-a review

Keywords: socioeconomic, prevention, micro endoscope, fluorescent; proflavine, cytoplasmic, processor, prospective, endoscopists

Abbreviations: ACS, american cancer society; WHO, world health organization; HRME, high-resolution micro-endoscope; PPV, positive predictive value; WLE, white light endoscopy; LCE, lugol chromoendoscopy

\section{Mini review}

Cancer continues to be one of the leading causes of morbidity and death globally. The latest statistics from the American Cancer Society (ACS) suggest 2 out of every 3 people diagnosed with cancer survive at least 5 years in the United States. ${ }^{1}$ The GLOBOCON project initiated by World health Organization (WHO) estimates approximately a cancer incidence of 14.1 million and 8.2 million deaths globally in 2012. In addition, the burden of the disease has shifted to less developed nations.2-5 Geographic variations stem from an array of factors including diet, lifestyle and trauma. Despite the implementation of endoscopic screening guidelines, the picture becomes more somber for low socioeconomic regions where access to diagnostic tools and public health prevention is limited, thus limiting their success. Suboptimal access to care, poor infrastructure, lack of funding and limited resources with respect to time and personnel amplify the challenges. ${ }^{2-5}$ Considering these constraints, costeffective, sensitive and efficient tools for early cancer detection would be invaluable for any healthcare setting faced with the pressures of limited resources. Early detection of cancer is critical to improving health outcomes. In most of cases, earlier detection translates into improved outcomes and survival. Current management practices require a tissue diagnosis prior to instituting cancer therapy because of the variable etiology of visible abnormalities. Infection, inflammation, trauma and anatomic variation can all mimic the appearance of a malignant or premalignant lesion, and the treatments for each of these conditions can vary dramatically. Similarly, a malignant focus may only show subtle surface changes and might be overlooked as a benign finding. New technologies have sought to overcome these challenges in diagnostic sensitivity and specificity; however they are not widely available, require specialized skill sets and are often cost-prohibitive. ${ }^{6}$

To address these issues, a novel, portable and cost-effective technology has been developed for the detection of epithelial cancers. High-Resolution Micro-Endoscope (HRME) is a unique technology that provides sub cellular resolution images after the use of a topical fluorescent agent. It consists of a fiber-optic probe with a light source, camera and tablet based processor. Topical application of fluorescent contrasting agent, specifically Proflavine $(0.01 \%)$, is used to illuminate sub cellular surfaces. An accessory channel of a standard endoscope is used to insert the HRME probe, and the $1 \mathrm{~mm}$ fiber optic micro-endoscope is placed on the mucosa providing a comprehensible picture of cellular features such as pleomorphism, nuclear cytoplasmic ratio, nuclear crowding. Image analysis software
Volume 4 Issue 2 - 2016

\section{Priyanka P, Jonathan Clavell-Hernandez, Comalita Lee, Nadeem N Dhanani \\ The University of Texas McGovern Medical School, USA}

Correspondence: Nadeem N Dhanani, The University of Texas McGovern Medical School, USA, Email pandhi.priyanka@gmail.com

Received: December 24, 2015 | Published: January 29, 2016

in addition to HRME assists physicians in differentiating benign from malignant lesions in real time by quantifying size of the nucleus. ${ }^{7,8}$ Therefore, HRME can serve as an optical biopsy tool for accurate in-vivo diagnostics that may allow real time decision making support during oncologic resection. This imaging modality has been the subject of multiple trials investigating its use in screening and surveillance of cancer of different origins including head and neck, gastrointestinal, esophageal, anal, cervical, bladder and also dental. HRME was first described by Muldoon et al.7 to demonstrate its imaging capabilities. Chang et al. ${ }^{9} 10$ achieved overall colorectal neoplasia sensitivity of 70 $\%(65 \%-76 \%)$, specificity $94 \%(87 \%-100 \%)$, and accuracy $85 \%$. Overall Inter-rater reliability between experts was $86 \%$ while between non-experts was $72 \%$. A prospective study by Parikh et al. ${ }^{11}$ concluded HRME to be an effective modality in differentiating neoplasia from non-neoplastic tissues. HRME achieved significantly higher specificity (95\%), positive predictive value (PPV, 87\%) and accuracy (94\%), compared with white light endoscopy (WLE) $(39 \%, 55 \%$, and $65 \%$ respectively). In addition, similar trends were observed for polyps (less than 10mm) compared to WLE in terms of accuracy (95\% vs. $64 \%)$, specificity $(98 \%$ vs. $40 \%)$ and PPV $(92 \%$ vs. $55 \%$ ). In a prospective, multi-center trial led by Protano et al. ${ }^{12}$ also observed HRME as an adjunct tool to Lugol chromoendoscopy (LCE) for improvement in the accuracy of esophageal squamous cell neoplasia screening and surveillance. Another study by Miles et al. ${ }^{13}$ targeting Otorhinology, head and neck cancers observed mean sensitivity, specificity, negative predictive value, positive predictive value, inter rater reliability respectively of $96 \%$ and $95 \%, 98 \%, 91 \%$, $81 \%$. In addition, further studies targeting efforts to train endoscopists to utilize this technique with high accuracy observed novice micro endoscopists had comparable sensitivity and slightly less specificity than experts. The learning curve to use and interpret HRME device and image by Parikh et al. ${ }^{14}$ found improvement in sensitivity from $49.3 \%$ to $97.3 \%$, specificity from $68.7 \%$ to $95.5 \%$. We at The University of Texas McGovern Medical School are evaluating the utilization of HRME for the first time for the early bladder cancer detection and expecting promising results (NCT02340650). 


\section{Conclusion}

HRME offers clinicians the ability to make immediate clinical decisions in real time at the point of care and expediting advanced healthcare delivery methods. Testing is under way to establish grading criteria that can offer valuable information in differentiating cancer from benign tissue, specifically for ambiguous appearing lesions. High inter-rater reliability also suggests the results to be reproducible, which can be difficult to achieve otherwise in disparate healthcare settings. Promoting targeted biopsies, conserving resources and improving efficiency of healthcare delivery in a reproducible model may render HRME a great tool in early cancer detection.

\section{Acknowledgements}

None.

\section{Conflicts of interest}

The authors declare that there is no conflict of interest.

\section{References}

1. http://www.cancer.org/cancer/index

2. Bray F, Ren JS, Masuyer E, et al. Global estimates of cancer prevalence for 27 sites in the adult population in 2008. Int J Cancer. 2013;132(2):1133-1145.

3. Ferlay J, Soerjomataram I, Dikshit R, et al. Cancer incidence and mortality worldwide: sources, methods and major patterns in GLOBOCAN 2012. Int $J$ Cancer. 2015;136(5):E359-E386.

4. Jemal A, Bray F, Center MM, et al. Global cancer statistics. CA Cancer J Clin. 2011;61(2):69-90.

5. Torre LA, Bray F, Siegel RL, et al. Global cancer statistics, 2012. CA Cancer J Clin. 2015;65(2):87-108.
6. Abu Dayyeh BK, Thosani N, Konda V, et al. ASGE Technology Committee systematic review and meta-analysis assessing the ASGE PIVI thresholds for adopting real-time endoscopic assessment of the histology of diminutive colorectal polyps. Gastrointest Endosc. 2015;81(3):502.

7. Muldoon TJ, Pierce MC, Nida DL, Williams MD, Gillenwater A, et al. (2007) Subcellular-resolution molecular imaging within living tissue by fiber microendoscopy. Opt Express. 15(25): 16413-16423.

8. Pierce M, Yu D, Richards KR. High-resolution fiber-optic microendoscopy for in situ cellular imaging. J Vis Exp. 2011;11:47.

9. Chang SK, Pavlova I, Marín NM, et al. Fluorescence spectroscopy as a diagnostic tool for detecting cervical pre-cancer. Gynecol Oncol. 2005;99(3):61-63.

10. Chang SS, Shukla R, Polydorides AD, et al. High resolution microendoscopy for classification of colorectal polyps. Endoscopy. 2013;45(7):553-559.

11. Parikh ND, Perl D, Lee MH, et al. In vivo diagnostic accuracy of highresolution microendoscopy in differentiating neoplastic from nonneoplastic colorectal polyps: a prospective study. Am J Gastroenterol. 2014;109(1):68-75.

12. Protano MA, Xu H, Wang G, et al. Low-Cost High-Resolution Microendoscopy for the Detection of Esophageal Squamous Cell Neoplasia: An International Trial. Gastroenterology. 2015;149(2):321329

13. Miles BA, Patsias A, Quang T, et al. Operative margin control with highresolution optical microendoscopy for head and neck squamous cell carcinoma. Laryngoscope. 2015;125(10):2308-2316.

14. Parikh ND, Perl D, Lee MH, et al. In vivo classification of colorectal neoplasia using high-resolution microendoscopy: Improvement with experience. J Gastroenterol Hepatol. 2015;30(7):1155-1160. 